Reprod. Nutr. Dévelop., 1986, 26 (1 B), 383-384.

\title{
Injections de doses physiologiques d'insuline chez la vache en lactation : effets sur les quantités ingérées et les métabolites sanguins
}

\author{
P. FAVERDIN
}

Station de Recherches sur la Vache laitière, I.N.R.A., St Gilles, 35590 L'Hermitage, France.

Summary. The intrajugular administration of $0.7 \mu \mathrm{g} / \mathrm{kg} \mathrm{BW}$ of insulin at the beginning of the meal depressed feed intake during the following $30 \min (-14 \%)$ when given to cows deprived of food for 11 hours but not in cows fed $4 \mathrm{~h}$ before. Thus, the role of insulin in the short-term control of cow feeding depends on nutritional status.

Chez le mouton, les injections d'insuline ont eu des effets nuls ou stimulants (Baile et Martin, 1971) sur la prise alimentaire à fortes doses, et dépressifs à faibles doses (Deetz et Wangsness, 1980). Dans cette étude, l'effet de doses physiologiques d'insuline sur l'ingestion a été mesuré après une période de jeûne et après un grand repas chez la vache en lactation.

Matériels et méthodes. Six vaches Pie-noires en milieu de lactation ont reçu par voie intra-jugulaire de l'insuline bovine $(0,35$ et $0,7 \mu \mathrm{g} / \mathrm{kg}$ de poids vif) ou du soluté physiologique, au cours de 2 essais indépendants.

Essai 1. - L'effet sur les quantités ingérées a été étudié avec des injections soit après une période de jeûne de $11 \mathrm{~h}$ (à $8 \mathrm{~h}$ au début du repas du matin), soit après un premier repas de quantité limitée (à $12 \mathrm{~h}$ ). Dans les 2 situations, les traitements ont été comparés dans un schéma en carré latin $3 \times 3$ avec 4 répétitions ( 2 groupes de 3 vaches $\times 2$ fois dans le temps). Les quantités ingérées ont été enregistrées en continu à l'aide d'auges montées sur capteurs électroniques et reliées à un micro-ordinateur.

Essai 2. - L'évolution de quelques composés sanguins (insuline, glucose, azote alpha aminé, acides gras non estérifiés (AGNE), sodium et potassium) a été mesurée chez 4 vaches au cours des 2 h suivant l'injection, celle-ci étant effectuée après une période de jeûne de $11 \mathrm{~h}$. Deux séries de mesures ont été réalisées ; dans la première les injections n'étaient pas suivies de repas afin de caractériser l'effet de l'insuline seule, alors que dans la seconde les animaux étaient alimentés aussitôt après l'injection.

Dans les 2 essais, la ration, constituée d'un mélange d'ensilage de maïs $(60 \%)$ et d'aliments concentrés $(40 \%)$, était offerte à volonté.

Résultats et discussion. L'injection de $0,7 \mu \mathrm{g} / \mathrm{kg}$ de poids vif d'insuline réalisée en début du grand repas chez des vaches non alimentées depuis $11 \mathrm{~h}$, entraîne une diminution de $14 \%$ des quantités ingérées $(P<0,05)$ durant la première demi-heure (fig. 1) qui est totalement compensée en fin de repas. La baisse de $7 \%$ avec la dose de $0,35 \mu \mathrm{g} / \mathrm{kg}$ n'est pas significative. Ce résultat confirme ceux de Deetz et Wangsness (1980) sur moutons montrant le rôle dépressif à 
court terme de l'insuline sur l'ingestion. Par contre, lorsque l'insuline est injectée à $12 \mathrm{~h}$, après que les animaux aient effectué un grand repas, l'évolution des quantités ingérées entre 12 et $21 \mathrm{~h}$ a été identique.
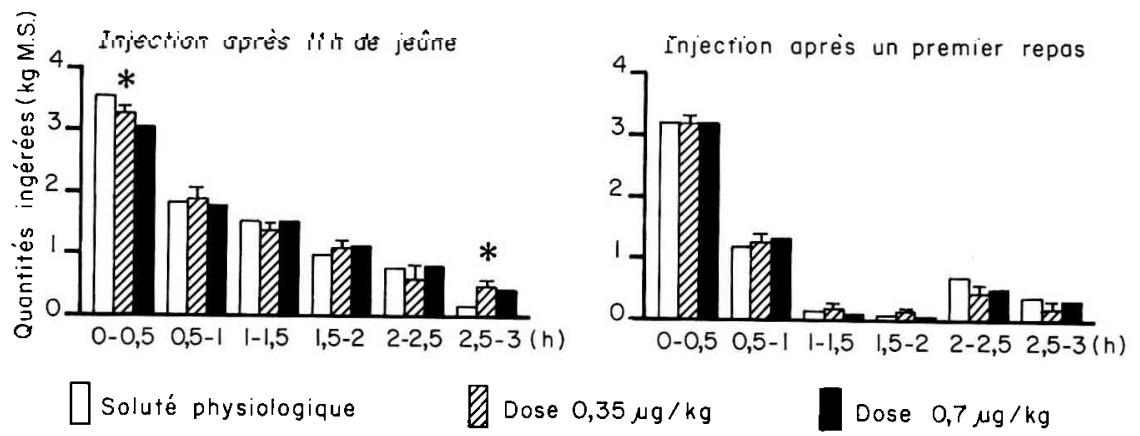

FIG. 1. - Influence de l'injection d'insuline sur les quantités ingérées au cours du repas chez la vache en lactation préalablement soumise à $11 \mathrm{~h}$ de jeûne ou ayant effectué un premier repas. ${ }^{*}=$ analyse de variance significative à $P<0,05$. Les quantités ingérées sont représentées $1 / 2 \mathrm{~h}$ par $1 / 2 \mathrm{~h}$ après l'injection. $T=$ écart-type de la moyenne (injection après $11 \mathrm{~h}$ de jeûne $\mathrm{n}=9$, après un premier repas $n=12)$.

L'insulinémie, maximale après l'injection, diminue ensuite très rapidement (demi-vie voisine de $7 \mathrm{~min}$ ) pour atteindre la valeur basale après environ $45 \mathrm{~min}$. Les teneurs plasmatiques de glucose, AGNE et potassium diminuent proportionnellement à la dose d'insuline entre 10 et 30 min après l'injection, celle de l'azote alpha aminé baisse plus tardivement (tabl. 1). Les effets métaboliques sont semblables chez les vaches recevant ou non un repas après l'injection d'insuline. La baisse de la glycémie après injection d'insuline intervient en même temps que celle de quantités ingérées.

TABL. 1. - Influence de l'injection d'insuline sur les principaux composés sanguins chez les vaches en lactation non alimentées depuis $11 \mathrm{~h}$.

\begin{tabular}{|c|c|c|c|c|}
\hline & \multirow{2}{*}{$\begin{array}{c}\text { Soluté } \\
\text { physiologique }\end{array}$} & \multicolumn{2}{|c|}{ Insuline } & \multirow{2}{*}{ E.T.M } \\
\hline & & $0,35 \mu \mathrm{g} / \mathrm{kg}$ & $0,7 \mu \mathrm{g} / \mathrm{kg}$ & \\
\hline Insuline $5 \min \left({ }^{1}\right)(\mathrm{ng} / \mathrm{ml})$ & $0,52 \mathrm{a}$ & $4,12 b$ & $7,44 \mathrm{c}$ & 0,52 \\
\hline Glucose $20 \mathrm{~min}(\mathrm{mg} / 100 \mathrm{ml})$ & 79,5 a & 61,8 b & 52,9 c & 1,3 \\
\hline Potassium $20 \min (\mathrm{mM} / \mathrm{l})$ & 4,2 a & $4,0 \quad b$ & $3,8 \mathrm{c}$ & 0,05 \\
\hline AGNE $30 \min (\mu \mathrm{M} / \mathrm{I})$ & $141 \quad a$ & $114 \quad b$ & $100 \quad b$ & 5,2 \\
\hline Azote aminé $60 \mathrm{~min}(\mathrm{mg} / 10 \mathrm{ml})$ & 31,6 a & $27,2 \quad b$ & 27,8 b & 1,0 \\
\hline
\end{tabular}

(1) Temps après l'injection. a, b, c : différences significatives à $P<0,05$.

AGNE = Acides gras non estérifiés. E.T.M. = Ecart-type de la moyenne $(n=4)$.

L'insuline injectée ne produisant un effet de satiété que lorsque le repas suit une période de jeûne, la sensibilité à l'insuline des centres régulateurs de l'ingestion dépend donc de l'état nutritionnel de l'animal.

Baile C. A., Martin F. H., 1971. Hormones and amino acids as possible factors in the control of hunger and satiety in sheep. J. Dairy Sci., 54, 897-905.

Deetz L. E., Wangsness P. J., 1980. Intrajugular administration of insulin on feed intake, plasma glucose and plasma insulin of sheep. J. Nutr., 110, 1976-1982. 